\title{
Nanohole Plasmons in Optically Thin
}

\section{Gold Films}

Tomas Rindzevicius, Yury Alaverdyan, Borja Sepulveda, Tavakol Pakizeh and Mikael Käll*

Department of Applied Physics, Chalmers University of Technology, SE-412 96 Göteborg, Sweden

Rainer Hillenbrand

Nano-Photonics Group, Max-Planck-Institut für Biochemie, 82152 Martinsried, Germany

Javier Aizpurua and F. Javier Garcia de Abajo Donostia International Physics Center,

Paseo Manuel de Lardizabal 4, Donostia-San Sebastian 20018, Spain

October 25, 2006 


\begin{abstract}
The optical properties of single nanoholes in optically thin $(t=20 \mathrm{~nm})$ gold films on glass have been studied experimentally and theoretically. The measured elastic scattering spectra from the nanoholes exhibit a broad resonance in the red part of the visible spectrum, which is qualitatively similar to localized surface plasmon (LSP) resonances in gold nanodisks. The hole resonance red-shifts with increasing hole diameter $(\mathrm{D}=60-107 \mathrm{~nm})$, similar to particle LSP resonances. These features could be well reproduced by electrodynamic simulations based on the Boundary Element Method (BEM). Further, the electric field distribution around the resonant nanoholes, obtained from the BEM simulations, exhibits a clear electric dipole pattern. This confirms the assignment of the hole resonance to a dipolar LSP resonance mode. However, in comparison to Au nanodisks of similar size, the hole LSP resonance exhibits a shorter dephasing time $(\tau)$. This observation can be understood in terms of an additional decay channel that is dominated by the short wavelength anti-symmetric bound $\left(\mathrm{a}_{b}\right)$ surface plasmon polariton (SPP) mode of the surrounding Au film. Experimental verification of the LSP-SPP coupling is obtained from near-field scanning optical microscopy images, which exhibit interference fringes due to SPP emission from the hole. The fringe periodicity corresponds to a SPP wavelength of $\lambda_{a_{b}} \approx 285 \mathrm{~nm}$, much less than both the free space wavelength $\lambda_{0}=633 \mathrm{~nm}$ or the wavelength of the more well-known symmetric leaky mode.
\end{abstract}

\footnotetext{
*To whom correspondence should be addressed. E-mail: kall@fy.chalmers.se
} 
Keywords: Nanohole optics, dark-field scattering, localized surface plasmons, surface plasmon polaritons, colloidal lithography.

\section{Introduction}

The discovery of enhanced optical transmission through sub-wavelength hole arrays [1] has attracted considerable interest in optical phenomena and applications associated with nanoscale apertures in metal films [2-9]. It is generally agreed that surface plasmon (SP) resonances play a key role in enhancing the transmission of light through apertures in noble metal films, although diffraction effects, especially in the presence of long-range order, also need to be considered $[10,11]$. Isolated nanoholes in optically thick metal films exhibit distinct optical properties [12] in comparison to nanohole arrays [13]. In order to make a clear distinction between diffraction and plasmonic coupling effects, a deeper understanding of single nanohole optical properties is needed. Zakharian et al. [14] recently presented a qualitative picture of the behavior of the electromagnetic fields around single elliptical apertures in optically thick metal films. It was argued that electric and magnetic dipoles are induced, and that these contribute significantly to the enhanced transmission. Such dipolar modes can be expected to result in localized surface plasmon (LSP) resonances, similar to those observed for noble metal nanoparticles [15]. However, the presence of the metal film obviously constitutes a major difference between a hole and a particle, because a nanohole LSP resonance can be expected to couple not 
only to ordinary light waves, but also to spatially extended surface plasmon polaritons (SPP's). Chang et al. [16] and Popov et al. [17] recently performed electrodynamic simulations which indicated that illumination of a nanohole in an optically thick gold film leads to polarization dependent SPP patterns, in good agreement with experiments using near-field scanning optical microscopy (NSOM) [18].

Concerning holes in optically thin metal films, the key role of surface plasmons has also been invoked [19]. We have previously demonstrated experimentally that isolated and short-range ordered arrays of circular nanoholes in $20 \mathrm{~nm}$ thick gold films on glass support excitations that are qualitatively very similar to particle LSP resonances [20]. Similar to particle plasmons, the nanohole resonance was shown to vary with hole diameter $D$, hole density and surrounding refractive index. Further, it was argued that these holes allow SPP excitation, which in turn renormalizes the nanohole resonance. However, we were not able to present rigorous experimental proof for LSP-SPP coupling and the assignment of the hole resonance to an electric dipole LSP mode remained speculative due to the considerable difficulty of performing high quality electrodynamic simulations for this type of nanooptical system. Despite these uncertainties, we were able to use the nanohole structures for advanced sensing applications [21,22]. Shortrange ordered nanoholes were utilized in biosensing experiments, in which it was possible to functionalize the inner $\mathrm{SiO}_{2}$ part of the holes with lipid bilayers for "biomimetic" surface plasmon resonance detection of molecular binding events [21]. This kind of measurement was even possible to perform using a single $60 \mathrm{~nm}$ diameter nanohole [22]. 
Utilizing alkanethiol self-assembled monolayers, we estimated that the spatial extension of the sensing region was 10 - $20 \mathrm{~nm}$, i.e. similar to what is expected for nanoparticle LSP's [22]. Still, the development of the nanohole structure into a competitive system for plasmonic bio/chemo sensing requires a more thorough understanding of hole plasmons in thin gold films.

In this paper, we focus on the fundamental optical properties of single nanoholes in optically thin gold films. We compare experimental elastic scattering spectra with simulated spectra obtained using the Boundary Element Method and find excellent agreement. This allows us to analyze the nature of the nanohole excitation through electromagnetic field distribution maps. Moreover, new NSOM data gives indisputable evidence for coupling to extremely short wavelength SPP's, which we identify as so called anti-symmetric bound modes of the thin Au film. The paper is organized as follows. Section II briefly describes the experimental methods used to prepare and study the single nanoholes. In Section III, the theoretical models that were employed to simulate the optical properties of the nanoholes are described, while Section IV discusses results and analyzes the LSP-SPP interaction in single nanoholes. The work is summarized in Section V.

\section{Experimental Methods}

The single nanoholes were prepared using colloidal lithography [23] on glass substrates.

First, colloidal white-sulphate polystyrene spheres (sulfate latex IDC USA) were elec- 
trostatically adsorbed on clean glass coverslips. A gold film (20 $\mathrm{nm}$ in thickness) is then thermally evaporated onto the colloidal sphere mask, after which the gold-capped spheres are removed by sonication in ethanol. The result is a $20 \mathrm{~nm}$ thick gold film containing circular holes with $\mathrm{SiO}_{2}$ at the bottom. Isolated single nanoholes $\left(\mathrm{d}_{\text {interhole }} \gtrsim\right.$ $8 \mu \mathrm{m})$ were prepared by kinetically interrupting particle adsorption at extremely low coverage. The diameters of the nanoholes are controlled by choosing the size of the spheres: for this study we prepared three different samples with average hole diameters 60, 76 and $107 \mathrm{~nm}$, as determined by scanning electron microscopy (SEM) imaging. The variation in hole diameters caused by sphere polydispersity was approximately \pm 10 nm. The optical properties of the isolated holes were quantified using elastic (Rayleigh) scattering, measured in a dark-field (DF) microscopy set-up. A standard inverted optical microscope (Nikon TE300) and an optical spectrometer (AvaSpec-2048) were used. As schematically depicted in Figure 1(a), unpolarized white light from a $100 \mathrm{~W}$ halogen lamp, $\mathrm{A}$, is focused on the sample, $\mathrm{B}$, using a dark-field condenser $(\mathrm{NA}=0.8-0.95$, $\theta \approx 53-72 \mathrm{deg})$. The scattered light is then collected with a $100 \mathrm{x}$ oil immersion objective $(\mathrm{NA}=0.5), \mathrm{C}$, and guided through a fiber to the spectrometer, D. For the objective/fiber combination used, the probe area has a diameter of $\sim 1.3 \mu \mathrm{m}$. Scattering spectra were obtained by first subtracting the background signal obtained from a hole-free region of the $\mathrm{Au}$ - film from the raw DF spectra, and then dividing by the white-light spectrum measured under bright-field conditions. Identical conditions were used for different hole samples, so that relative scattering intensities could be determined. Figure 1(b) and (c) 
show typical SEM and DF images of single holes.

To complete the experimental methodology, near-field optical imaging of a single 60 nm hole was performed by a scattering-type scanning near-field optical microscope (sSNOM) [24] that employs Pt coated Si tips as light scattering probes. The tip is illuminated from the side by a TM polarized HeNe laser beam $(\lambda=633 \mathrm{~nm})$. The illumination angle of the slightly focused laser beam is about $40^{\circ}$, measured from the gold surface, and the laser beam illuminates the hole from the air side. The light backscattered from the tip is detected with a heterodyne interferometer yielding amplitude and phase.

\section{Theoretical Methods}

Near-field calculations for nanoholes were performed using BEM [25,26]. The electric and magnetic fields are written in terms of scalar and vector potentials $\phi$ and $\mathbf{A}$, and the solution to Maxwell's equations that vanishes away from the hole is expressed in terms of a distribution of charges $\sigma_{j}$ and currents $\mathbf{h}_{j}$ that are defined on either side of all boundaries between the different media. In our case, $j=1,2$, and 3 will label vacuum, gold, and glass, respectively. For $\mathbf{r}$ inside medium $j$, one has

$$
\phi(\mathbf{r})=\phi^{e}(\mathbf{r})+\int_{S_{j}} d \mathbf{s} G_{j}(|\mathbf{r}-\mathbf{s}|) \sigma_{j}(\mathbf{s})
$$

and

$$
\mathbf{A}(\mathbf{r})=\mathbf{A}^{e}(\mathbf{r})+\int_{S_{j}} d \mathbf{s} G_{j}(|\mathbf{r}-\mathbf{s}|) \mathbf{h}_{j}(\mathbf{s}),
$$


where $S_{j}$ refers to the boundary of medium $j$;

$$
G_{j}(|\mathbf{r}-\mathbf{s}|)=\frac{e^{i k_{j}|\mathbf{r}-\mathbf{s}|}}{|\mathbf{r}-\mathbf{s}|}
$$

is the scalar-wave Green function propagator in medium $j ; k_{j}=(\omega / c) \sqrt{\epsilon_{j}}$ is the momentum of light inside medium $j$ of permittivity $\epsilon_{j}$; and $\phi^{e}$ and $\mathbf{A}^{e}$ are scalar and vector potentials associated with the incoming light, which are non-vanishing only for $j=1$ (i.e., in the medium above the film, which is illuminated by an incoming plane wave). The boundary conditions of the electromagnetic field are then imposed and yield a set of self-consistent surface integral equations that determine the surface sources and that are solved by linear algebra techniques upon discretization of the integrals [26]. The BEM relies on parametrization of finite structures. The near field is thus calculated for films of finite increasing size until convergence is achieved.

The far-field optical response is, however, extremely sensitive to the termination of the film. We have therefore extended the BEM to cope with infinite planar interfaces. In our scheme, the BEM is used to parametrize a hole-like void inside the metal and to calculate forward and backward scattering coefficients for planar and evanescent waves incident from above and from below the hole. These coefficients are regarded as elements of a scattering matrix. The film boundaries lead to diagonal scattering matrices for incident plane waves, whose elements are given by Fresnel's reflection and transmission coefficients. All of these elements (the hole and the film boundaries) are combined into a multiple-scattering scheme, producing the full scattering matrix of the film with the 
hole-like inclusion. The film boundaries are made increasingly close to the hole until the actual hole-in-a-film structure is realized. Convergence with increasing number of parametrization points in the underlying BEM calculation, with increasing number of planar and evanescent waves, and with decreasing distance between the film boundaries and the hole has been checked. We are interested in the matrix elements that connect the incoming light with transmitted waves. The latter are integrated over all transmission directions, so that the resulting transmission intensity can be compared with dark-field scattering measurements.

In addition to the BEM simulations, we calculated the SPP characteristics for a homogeneous planar $20 \mathrm{~nm}$ gold film between glass and air. The calculation of the in-plane wavevector and field distribution of the anti-symmetric surface plasmon polariton has been carried out using a transfer matrix formalism [27]. Such a formalism allows to relate the tangential components of the electromagnetic fields at the two sides of the metallic layer. Since SPP's are transversal magnetic (TM) waves, only the TM field components are required [27]. To calculate the dispersion of the bound anti-symmetric mode, we assume that there are no incident plane waves from the surrounding dielectrics. With this condition, a homogeneous system of equations for the amplitudes of the TM field components at both sides of the metal is obtained. The non-trivial solution of the system generates an equation, which solution provides the in-plane wavevector $(k)$ of the bound mode. Once $k$ is calculated, the electromagnetic field distribution in each medium can be determined. 
In order to find the complex propagation constants of leaky modes in this lossy waveguide, the reflection pole method was implemented [28]. The technique is based on the transfer matrix method and it monitors the phase of the reflection coefficient denominator. A numerical differentiation of the phase of the reflection coefficient denominator vs. the effective index produces Lorentzian-type peaks (real part of the normalized mode propagation constant). The half width at half maximum of these peaks is equal to the normalized imaginary part of the mode propagation constants.

\section{Results and Discussion}

\subsection{Optical Properties of Isolated Nanoholes}

Using the dark-field spectroscopy set-up, it is relatively straightforward to identify individual nanoholes and measure elastic scattering spectra, see insets in Figure 1(b) and (c). Figure 2(a) shows scattering spectra averaged over 30-40 single nanoholes for each of the three diameters. The spectra are inhomogeneously broadened because of the spread in polystyrene sphere sizes, but the effect is not significant due to the intrinsically large line-width of the individual hole spectra. Consequently, the averaged spectra are well described by Lorentzian line-shapes without any Gaussian (inhomogeneous) contribution. The averaged peak positions extracted from the Lorentzian curve fits are 1.80, 1.70 and $1.57 \mathrm{eV}$ for 60,76 and $107 \mathrm{~nm}$ holes, respectively. The hole resonance thus red-shifts 
monotonously with increased hole diameter, see inset of Figure 2(a), in agreement with our earlier investigations [20] and qualitatively similar to gold nanodisks [29]. However, the nanohole spectrum is red-shifted and broader in comparison with that of a nanodisk of identical size.

In a simplified analysis, the LSP resonance position for a nanodisk is determined by the real part of a dipole polarizability denominator, which primarily depends on the disk aspect ratio [30]. However, to the best of our knowledge, an equivalent electrostatic point dipole model for a hole in a thin film has not been formulated. Consequently, the single hole problem has to be treated by rigorous electrodynamic methods that give the solution to Maxwell's equations. In this work, this is done using the BEM. The gold dielectric function was taken from Johnson and Christy [31]. Figure 2(b) shows the calculated transmission spectra of 60 and $107 \mathrm{~nm}$ nanoholes. To illustrate the large increase in the transmission cross section for larger nanoholes, the spectra in Figure 2(b) were normalized to the geometrical cross-section. The calculated transmission peak positions are $1.95 \mathrm{eV}$ for $\mathrm{D}=60 \mathrm{~nm}$ and $1.65 \mathrm{eV}$ for $\mathrm{D}=107 \mathrm{~nm}$ single holes, in excellent agreement with the experimental spectra. This is a good indication that also the nearfield properties of the nanohole resonance can be analyzed using the BEM, as will be discussed below.

As can be seen in Figure 2(a), an increase in hole diameter not only leads to a red-shift, but also causes spectral broadening, i.e. the lifetime of the hole resonance is shortened. This is in contrast to gold nanodisks, for which the LSP lifetime is not significantly 
affected by a change in a disk diameter from $\mathrm{D}=60$ to $120 \mathrm{~nm}$ (see inset in Figure 2(c)) [29]. To illustrate the spectral broadening effect in more detail, we show the relationship between the resonance energy and the full width at half maximum $(\Gamma)$ for a large number of different nanoholes in Figure 2(c). In this plot, we utilize the spread in individual hole diameters, and thus in resonance position, in the three samples to access a more continuous range of resonance parameters. The plot clearly indicates a more or less linear relationship, i.e. $\Gamma \approx 1.96-0.77 \cdot \mathrm{E}_{\text {res }}[\mathrm{eV}]$, even within each sample. For 60 and $107 \mathrm{~nm}$ nanoholes, the average dephasing times, which are related to the resonance widths through $\Gamma=2 \hbar / \tau$, are 2.4 and 1.7 fs, respectively, which can be compared to $\tau \approx 2.2$ fs for disks of $\mathrm{D}=60-120 \mathrm{~nm}$. The difference is most notable for the larger diameters, where both the hole and the disk resonances are well bellow the interband region of gold, i.e. $\tau_{\text {disk }} \approx 1.3 \tau_{\text {hole }}$ for $\mathrm{D} \approx 110 \mathrm{~nm}$.

\subsection{Electric Field Distribution Around Single Nanoholes}

In order to further our understanding of the nature of the resonant hole excitations, the near-field distribution around single holes excited at resonance are necessary, see Figure 3. The BEM plots were made at $1.96 \mathrm{eV}$ for the $\mathrm{D}=60 \mathrm{~nm}$ hole (Figure 3(a)-(c)) and at $1.65 \mathrm{eV}$ for the $\mathrm{D}=107 \mathrm{~nm}$ hole (Figure $3(\mathrm{~d})-(\mathrm{f})$ ), i.e. at energies corresponding to the scattering maxima of Figure 2 (b). At a distance of $5 \mathrm{~nm}$ above the metal surface, the E-field distribution exhibits clear electric dipole patterns for both hole sizes, Figure 
3(b) and (e). If the E-field is plotted $20 \mathrm{~nm}$ above the nanoholes instead, the dipolar pattern is lost more quickly for the smaller hole, compare Figure 3(a) and (d), similar to the case of plasmonic nanoparticles, for which the spatial extension of the dipolar near-field component is essentially determined by particle size [32]. The maximum Efield enhancement is observed close to the hole edges, i.e. $|E| /\left|E_{0}\right|=4.1$ and 5.5 for the 60 and $107 \mathrm{~nm}$ holes, respectively, because it is at these positions that the charge accumulation associated with the dipolar excitation is the largest. The calculations are thus fully consistent with the idea that the hole resonance can be viewed as a localized electric dipole plasmon, i.e. a "hole LSP". However, the E-field enhancement around the nanoholes is small in comparison with nanodisks [33] and nanorings [34] of similar dimensions, but this is not surprising, considering the lower quality factor of the hole resonance compared to disk or ring LSP's.

The near-field plots can also be used to estimate the decay length of the E-field inside the holes, which is an important parameter in e.g. biosensing applications [21,22]. If the field decay is assumed to be exponential, i.e. $E(r) \propto E_{0}(\exp (x / \delta)+\exp (-x / \delta))$, we obtain decay length of the order of $\sim 30$ and $\sim 40 \mathrm{~nm}$ in the $\mathrm{x}$-direction towards the center of the hole for the 60 and $107 \mathrm{~nm}$ holes, respectively. This is in reasonable agreement with experimental data for $60 \mathrm{~nm}$ holes, which yielded a surface-averaged value of $\delta \approx 10-20 \mathrm{~nm}[22]$. 


\subsection{Generation of SPP's by Single Nanoholes}

To study possible LSP-SPP coupling effects in more detail, we used scattering type nearfield scanning optical microscopy. This experiment confirmed that the nanoholes indeed act as point sources of SPP's, see Figure 4(a),(b). The measurement is qualitatively similar to NSOM experiments on isolated holes in optically thick gold films [18]. As can be seen from the figures, the emanating SPPs are more pronounced in the direction of electric field polarization, and the cone-like SPP fringes have different periods across the $x$ axis (defined as the projection of the incident wavevector along the surface), see Figure 4(c).

Close to the hole, the near-fields at the metal tip disturb the LSP. For this reason, we do not consider the immediate vicinity of the hole in the following and adjust the grey-scale for optimal visibility of the fringes further away from the hole. Similar to surface phonon polariton probing [35], the fringes far from the hole can be explained by interference of the incoming field $E_{i}$ with the surface plasmon polariton wave $E_{S P P}$, see Figure $4(\mathrm{~d})$. The signal amplitude we detect, $E_{t}$, is proportional to the coherent sum of the incident field and the surface plasmon field, i.e. $E_{t}=E_{i}+E_{S P P} \propto\left(1+A \cdot \exp \left[i\left(k_{S P P}-k \cos (\theta)\right) x+\right.\right.$ $\left.\varphi_{0}\right]$ ), where $\mathrm{A}$ is the $\mathrm{SPP}$ field amplitude relative to the incoming field $E_{i}, k_{S P P}$ is the $\mathrm{SPP}$ wavevector, and $\varphi_{0}$ is the phase of the SPP relative to the incoming field $E_{i}$ at the source. The fringe spacing in the forward direction is then $2 \pi /\left(k_{S P P}-k \cos (\theta)\right)$, which is larger than it is in the opposite $(-\mathrm{x})$ direction, $2 \pi /\left(k_{S P P}+k \cos (\theta)\right)$. The SPP wavelength 
estimated from the fringe periodicity in the $+\mathrm{x}$ and $-\mathrm{x}$ propagation directions was found to be $304 \pm 10 \mathrm{~nm}$ and $266 \pm 10 \mathrm{~nm}$, respectively. The average value, $\lambda_{S P P} \approx 285 \mathrm{~nm}$, is less than half the free space wavelength, which rules out classical diffraction effects as the source of the fringe pattern.

Figure 5(a) shows the calculated SPP dispersion relations for a $20 \mathrm{~nm}$ Au film on glass $\left(\mathrm{n}_{\text {glass }}=1.52\right)$ in air $\left(\mathrm{n}_{\text {air }}=1.00\right)$ within the energy range of interest. We consider here the Fano modes that are physically admissible [36], i.e the symmetric leaky ( $\left.{ }_{l}\right)$ and the anti-symmetric bound $\left(a_{b}\right)$ modes. The film only supports one bound mode, as the gold film thickness is below the cut-off $(\approx 50 \mathrm{~nm})$ for the symmetric bound mode [36]. As can be seen from the figure, the dispersion curve for the $a_{b}$ mode is well below the light-lines in either media, i.e. the SPP wavelength is much shorter than for any freespace light-waves of the same energy. This means that direct excitation of the $\mathrm{a}_{b}$ mode, for example via prism coupling, is prohibited and the SPP mode can only be launched through defect coupling.

The SPP wavelength of the $a_{b}$ mode at the free space wavelength of $\lambda=633 \mathrm{~nm}$ is found to be $\sim 308 \mathrm{~nm}$, which is in good agreement with the value $\lambda_{S P P} \approx 285 \mathrm{~nm}$ estimated from the s-NSOM experiment (see "+" symbol in Figure 5(a)). We note that the $a_{b}$ mode is characterized by a charge displacement pattern that consists of equal sign charges on the two interfaces [37], which results in an anti-symmetric field distribution, see Figure 5(b). The charge distribution around the hole thus coincides with that of an electric dipole, which explains why the hole LSP mode can couple to the $\mathrm{a}_{b}$-SPP mode. In addition to 
the $\mathrm{a}_{b}$ mode, the structure also supports the $\mathrm{s}_{l}$ mode. In this case, charges of opposite sign occurs at the two interfaces, resulting in a symmetric field distribution, see Figure 5(b). The $\mathrm{s}_{l}$ mode should therefore not couple to a dipolar hole plasmon.

From the results above, the most plausible explanation of why the hole resonance has a shorter life-time than the corresponding disk LSP is simply that it has access to an additional decay channel in the form of surface plasmon polaritons. Calculations of the energy dissipation of a dipole emitter close to a semi-infinite Au slab (not shown) indicates that the quantum efficiency for SPP generation, i.e. the probability that an emitted photon launches a SPP wave rather than propagate to the far field, is of the order $50 \%$ for the energy range of interest here [38]. Although the LSP-SPP coupling strength will depend sensitively on the detailed geometry of the hole-film system, similar to the case of a spherical metal particle outside a thin metal film [39], it is clear that SPP interactions will have a large impact on the life-time of any localized resonance with an energy that overlaps with the SPP density of states (DOS). In a solid state physics picture, the broadening caused by LSP-SPP coupling corresponds to the imaginary part of a LSP self-energy, while the real part corresponds to a frequency shift. The DOS of the relevant SPP mode (the $\mathrm{a}_{b}$-mode) peaks at high energies $(\sim 2.3 \mathrm{eV})$, and we therefore expect that the hole LSP mode is pushed towards lower energies compared to the LSP of a disk of the same size, which is not renormalized by LSP-SPP coupling. This picture can thus qualitatively explain why hole LSP's are red-shifted compared to disks. 


\section{Summary}

In this paper, we have studied the optical properties of single nanosized holes prepared by colloidal lithography in optically thin $(20 \mathrm{~nm})$ gold films. The holes exhibit a resonance that red-shifts and broadens when the hole diameter is increased. These features could be well reproduced by electrodynamic simulations based on the Boundary Element Method. The calculated E-field distribution around the nanoholes displayed a clear electric dipole character, which supports our previous assignment of the hole resonance to a localized surface plasmon resonance.

The shorter dephasing time of the hole LSP compared to disk plasmons was interpreted as an effect of LSP-SPP coupling. This picture was supported by near-field microscopy of an isolated $60 \mathrm{~nm}$ nanohole, which showed that the hole indeed acts as a scattering center for SPP's. The fringe period obtained from the s-NSOM image was found to be $\sim 285 \mathrm{~nm}$ for an illumination wavelength of $633 \mathrm{~nm}$, which agrees with the wavelength of the anti-symmetric bound SPP mode of a homogeneous $20 \mathrm{~nm}$ Au film on glass.

\section{Acknowledgments}

Funding for this work was provided by the Swedish Research Council and the Swedish Foundation for Strategic Research. We would like to thank George C. Schatz, William L. Barnes, Peter Nordlander, W. Andrew Murray, Joan Alegret and Rashid Zia for stimulating discussions and suggestions. 


\section{References}

[1] Ebbesen, T. W.; Lezec, H. J.; Ghaemi, H. F.; Thio, T.; Wolff, P. A. Nature 1998, 391, 667-669.

[2] Kim, T. J.; Thio, T.; Ebbesen, T. W.; Grupp, D. E.; Lezec, H. J. Opt. Lett. 1999, $24,256$.

[3] Lezec, H. J.; Degiron, A.; Devaus, E.; Linke, R. A.; Martin-Moreno, L.; GarciaVidal, F. J.; Ebbesen, T. W. Science 2002, 297, 820-822.

[4] Levene, M. J.; Korlach, J.; Turner, S. W.; Foquet, M.; Craighead, H. G.; Webb, W. W. Science 2003, 299, 682-686.

[5] Devaux, E.; Ebbesen, T. W.; Weeber, T. W.; Dereux, J.-C. A. Appl. Phys. Lett. 2003, 83, 4936-4938.

[6] Brolo, A. G.; Gordon, R.; Leathem, B.; Kavanagh, K. L. Langmuir 2004, 20, $4813-4815$.

[7] Brolo, A. G.; Arctander, E.; Gordon, R.; Leathem, B.; Kavanagh, K. L. Nano. Lett. 2004, 4, 2015-2018.

[8] Williams, S. M.; Rodriguez, K. R.; Teeters-Kennedy, S.; Stafford, A. D.; Bishop, S. R.; Lincoln, U. K.; Coe, J. V. J. Phys. Chem. B 2004, 108, 11833-11837.

[9] Barnes, W. L.; Dereux, A.; Ebbesen, T. W. Nature 2003, 424, 824-830. 
[10] Lezec, H. J.; Thio, T. Optics Express 2004, 12, 3629-3651.

[11] Garcia de Abajo, F. J. Optics Express 2002, 10, 1475-1484.

[12] Degiron, A.; Lezec, H. J.; Yamamoto, N.; Ebbesen, T. W. Opt. Commun. 2004, 239, 61-66.

[13] Degiron, A.; Ebbesen, T. W. J. Opt. A: Pure Appl. Opt. 2005, 7, S90-S96.

[14] Zakharian, A. R.; Mansuripur, M.; Moloney, J. V. Opt. Express 2004, 12, 26312648.

[15] Hillenbrand, R.; Keilmann, F.; Hanarp, P.; Sutherland, D. S.; Aizpurua, J. Appl. Phys. Lett. 2003, 83, 368-370.

[16] Chang, S. H.; Gray, S. K.; Schatz, G. C. Opt. Express 2005, 13, 3150-3165.

[17] Popov, E.; Neviere, M.; Boyer, P.; Bonod, N. Opt. Commun. 2005, 255, 338-348.

[18] Yin, L.; Vlasko-Vlasov, V. K.; Rydh, A.; Pearson, J.; Welp, U.; Chang, S. H.; Gray, S. K.; Schatz, G. C.; Brown, D. B.; Kimball, C. W. Appl. Phys. Lett. 2004, 85, 467-469.

[19] Wannemacher, A. Opt. Commun. 2001, 195, 107-118.

[20] Prikulis, J.; Hanarp, P.; Olofsson, L.; Sutherland, D.; Käll, M. Nano Lett. 2004, 4, $1003-1007$. 
[21] Dahlin, A.; Zäch, M.; Rindzevicius, T.; Käll, M.; Sutherland, D.; Höök, F. J. Am. Chem. Soc. 2005, 127, 5043-5048.

[22] Rindzevicius, T.; Alaverdyan, Y.; Dahlin, A.; Höök, F.; Sutherland, D.; Käll, M. Nano Lett. 2005, 5, 2335-2339.

[23] Hanarp, P.; Sutherland, D. S.; Gold, J.; Kasemo, B. Colloids and Surfaces A: Physicochem. Eng. Aspects 2003, 214, 23-36.

[24] Keilmann, F.; Hillenbrand, R. Phil. Trans. R. Soc. Lond. A 2004, 362, 787-805.

[25] Garcia de Abajo, F. J.; Howie, A. Phys. Rev. Lett. 1998, 80, 5180.

[26] Garcia de Abajo, F. J.; Howie, A. Phys. Rev. B 2002, 65, 115418.

[27] Sepulveda, B.; Lechuga, L. M.; Armelles, G. J. Lightw. Technol. 2006, 24, 945-955.

[28] Anemogiannis, E.; Glytsis, E.; Gaylord, T. K. J. Lightw. Technol. 1999, 17, 929941.

[29] Hanarp, P.; Käll, M.; Sutherland, D. S. J. Phys. Chem. B 2003, 107, 5768-5772.

[30] Bohren, C. F.; Huffman, D. R. Absorption and scattering of light by small particles; John Wiley \& Sons, 1983.

[31] Johnson, P. B.; Christy, R. W. Phys. Rev. B 1972, 6, 4370-4379. 
[32] Gunnarsson, L.; Rindzevicius, T.; Prikulis, J.; Kasemo, B.; Käll, M.; Zou, S.; Schatz, G. C. J. Phys. Chem. B 2005, 109, 1079-1087.

[33] Kelly, K. L.; Coronado, E.; Zhao, L. L.; Schatz, G. C. J. Phys. Chem. B 2003, 107, 668-677.

[34] Aizpurua, J.; Hanarp, P.; Sutherland, D. S.; Käll, M.; Bryant, G. W.; Garcia de Abajo, F. J. Phys. Rev. Lett. 2003, 90, 057401-1.

[35] Huber, A.; Ocelic, N.; Kazantsev, D.; Hillenbrand, R. Appl. Phys. Lett. 2005, 87, 081103.

[36] Burke, J. J.; Stegeman, G. I.; Tamir, T. Phys. Rev. B 1986, 33, 5186-5201.

[37] Sernelius, B. E. Surface Modes in Physics; Wiley, 2001.

[38] Garcia de Abajo, F. J. et al., in preparation.

[39] Le, F.; Lwin, N. Z.; Steele, J. M.; Käll, M.; Halas, N. J.; Nordlander, P. Nano Lett. 2005, 5, 2009-2013. 


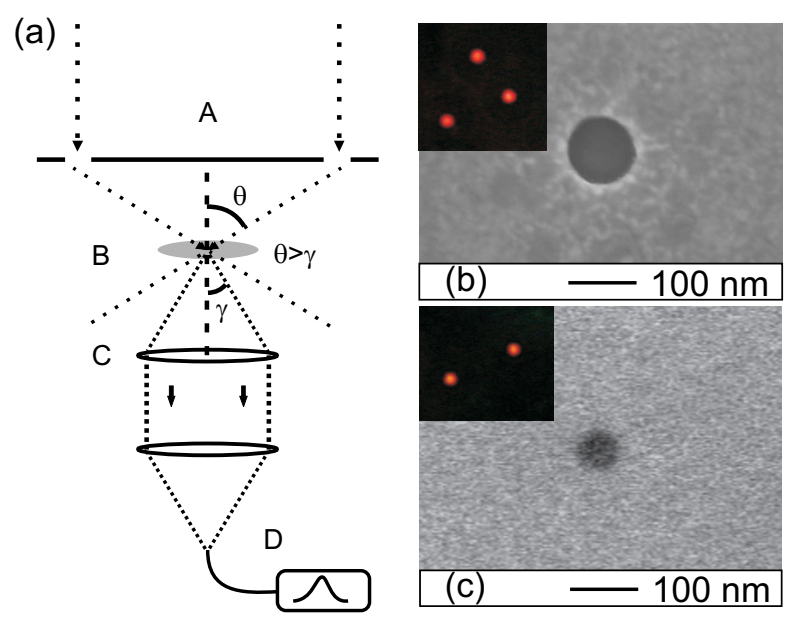

Figure 1: (a) - Schematics of the dark-field (DF) set-up, (b) and (c) - representative SEM images of single $\sim 107$ and $\sim 60 \mathrm{~nm}$ nanoholes in $20 \mathrm{~nm}$ thick gold film, respectively.

The insets show corresponding single hole images under DF illumination. 

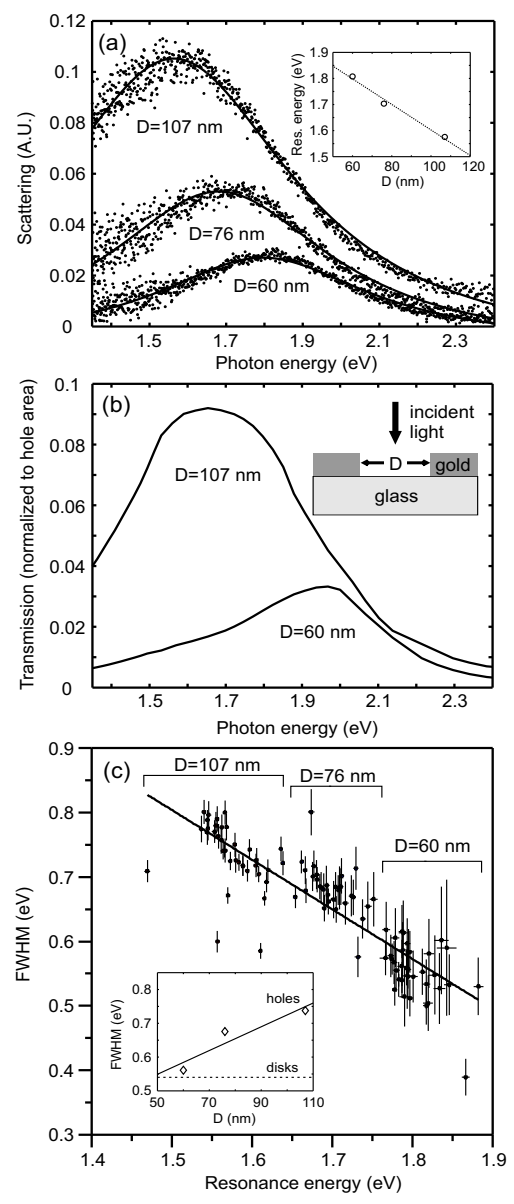

Figure 2: (a) Averaged elastic scattering spectra of 30-40 isolated nanoholes per sample with average diameter of 60, 76 and $107 \mathrm{~nm}$. The spectra are fitted to Lorentzian functions. The inset shows the variation in average resonance energy versus average hole size. (b) Calculated intensity transmitted to the half-space below the hole (see inset) normalized to hole area for 60 and $107 \mathrm{~nm}$ holes. (c) Correlation between resonance energy and the full width at half maximum (FWHM) for the whole ensemble of isolated holes. Inset shows peak width vs. hole diameter together with the trend reported for $\mathrm{Au}$ nanodisks of similar sizes according to ref. [29]. 

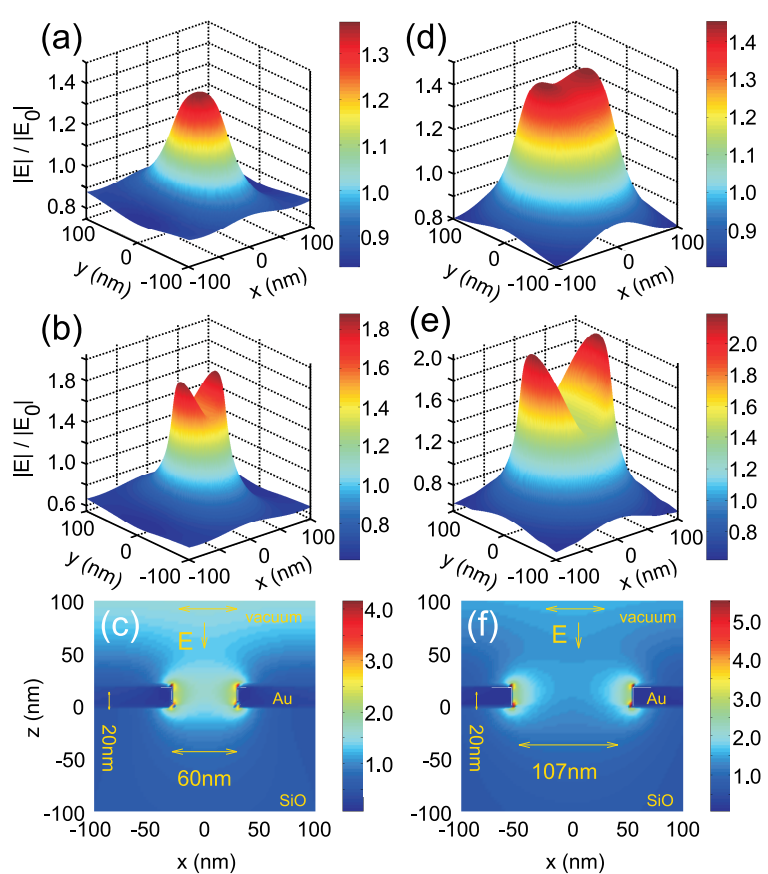

Figure 3: Calculated E-field distribution around $60 \mathrm{~nm},(\mathrm{a})-(\mathrm{c})$, and $107 \mathrm{~nm},(\mathrm{~d})-(\mathrm{f})$, holes in a $20 \mathrm{~nm}$ thick $\mathrm{Au}$ film on $\mathrm{SiO}_{2}$. The near-field plots were made $20 \mathrm{~nm},(\mathrm{a})$, (d), and $5 \mathrm{~nm},(\mathrm{~b}),(\mathrm{e})$, above the hole. The calculations were produced using the BEM, and the normally incident monochromatic light was polarized in the $x$ direction. 

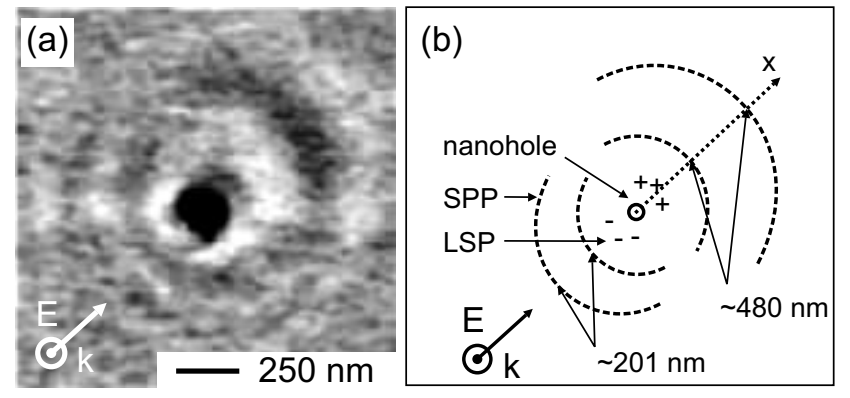

(c)
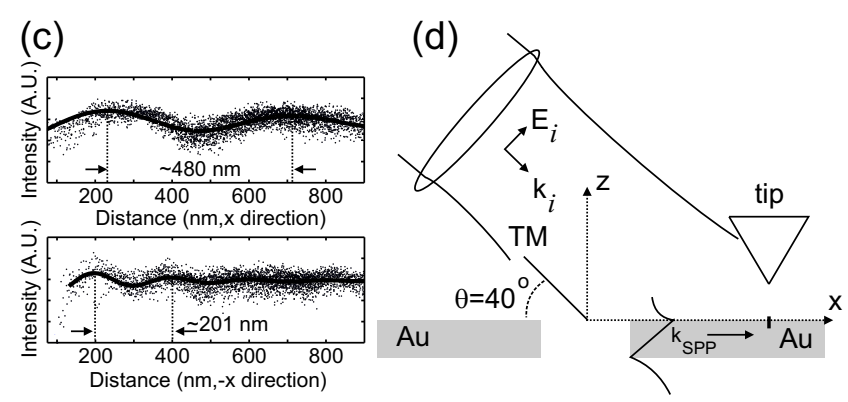

Figure 4: (a) Scattering-type near-field optical image of a single $60 \mathrm{~nm}$ nanohole on glass taken with a $40^{\circ}$ illumination angle and $\lambda=633 \mathrm{~nm}$. The beam is TM polarized. (b) Schematic illustration of SPP excitation around a nanohole. (c) Scattering intensity as a function of distance from the hole averaged over a cone with $90^{\circ}$ opening angle along the x-axis. The data was fitted to the expression for the signal amplitude, $E_{t}$. (d) Side-view of the s-NSOM experiment. 

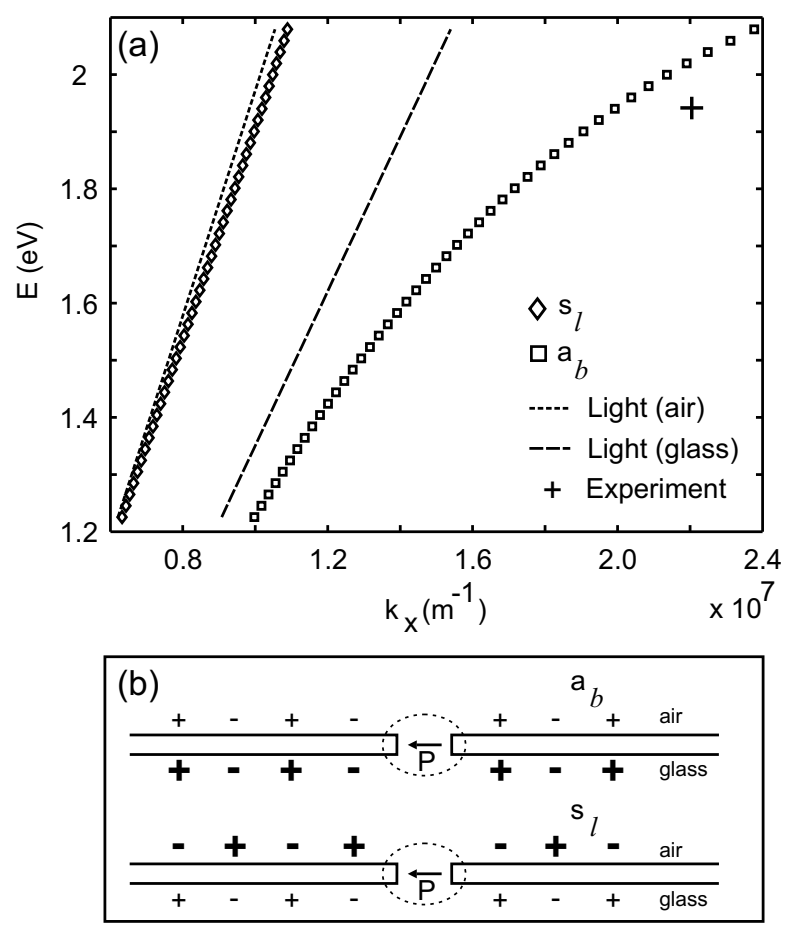

Figure 5: (a) Dispersion relations for the the anti-symmetric bound $\left(\mathrm{a}_{b}\right)$ and the symmetric leaky $\left(\mathrm{s}_{l}\right)$ modes in a $20 \mathrm{~nm}$ thick gold film on a glass substrate in air obtained using the reflection pole method. The dashed lines show the light lines at grazing incidence in the two media. The "+" symbol is the experimental value of $\mathrm{k}_{S P P}$ obtained from the Figure 4(a). (b) Illustration of a dipole moment created by a nanohole, $\mathrm{P}$, and the charge distributions created by the $\mathrm{a}_{b}$ and the $\mathrm{s}_{l}$ modes. 